\title{
The Effect of S-Adenosylmethionine Treatment on Neurobehavioral Phenotypes in Lesch-Nyhan Disease: A Case Report
}

\author{
Ken Momosaki Jun Kido $^{\mathrm{a}}$ Shiro Matsumoto ${ }^{\mathrm{a}}$ Atsuo Taniguchi ${ }^{\mathrm{b}}$

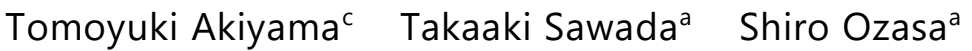 \\ Kimitoshi Nakamura ${ }^{a}$ \\ aDepartment of Pediatrics, Graduate School of Medical Sciences, Kumamoto University, \\ Kumamoto, Japan; 'Institute of Rheumatology, Tokyo Women's Medical University, \\ Tokyo, Japan; 'Department of Child Neurology, Okayama University Hospital, \\ Okayama, Japan
}

\section{Keywords}

Dystonia $\cdot$ g.151C > T Laryngeal stridor $\cdot$ Lesch-Nyhan disease $\cdot S$-adenosylmethionine $\cdot$

Self-injury

\begin{abstract}
Lesch-Nyhan disease (LND) is an X-linked recessive disorder caused by a deficiency in hypoxanthine-guanine phosphoribosyl transferase. Patients with LND experience involuntary movements, including dystonia, choreoathetosis, opisthotonos, ballismus, and self-injury. Alleviating these involuntary movements is important to improve the quality of life in patients with LND. Many clinicians have difficulty controlling these involuntary movements in their patients, and there are no established and effective treatments. A 6-month-old boy with LND presented with generalized dystonia and self-injury behavior that was alleviated after receiving S-adenosylmethionine (SAMe). His self-injury behavior completely resolved after he received SAMe and
\end{abstract}




\section{Case Reports in Neurology}

Case Rep Neurol 2019;11:256-264

DOI: $10.1159 / 000502568$

2019 The Author(s). Published by S. Karger AG, Basel www.karger.com/crn

Momosaki et al.: The Effect of S-Adenosylmethionine Treatment on Neurobehavioral Phenotypes in Lesch-Nyhan Disease: A Case Report

risperidone. Although he had often experienced inspiratory stridor because of laryngeal dystonia and frequently developed aspiration pneumonitis and bronchitis, no inspiratory stridor was noted after SAMe treatment. The patient is continuing to receive SAMe and risperidone. SAMe treatment alleviates dystonic movements and improves quality of life in pediatric patients with LND. Additional research is needed to determine the long-term safety and efficacy of SAMe and its appropriate dosage.

(C) 2019 The Author(s)

Published by S. Karger AG, Basel

\section{Introduction}

Lesch-Nyhan disease (LND) (Online Mendelian Inheritance in Man \# 308000) is an Xlinked recessive disorder caused by a deficiency in hypoxanthine-guanine phosphoribosyl transferase (HPRT, Enzyme Commission \# 2.4.2.8). The disease occurs in approximately $1 / 380,000$ live births in Canada and 1/235,000 live births in Spain [1], although its incidence in Japan is unknown. Its primary characteristics include hyperuricemia and neurological manifestations, including global developmental delay. The clinical phenotype presents mainly in men, but there are also female patients with LND.

Patients with LND suffer from involuntary movements, including dystonia, choreoathetosis, opisthotonus, ballismus, and self-injury. Alleviating these involuntary movements is important for improving quality of life. The underlying cause of the neurobehavioral phenotypes of LND is decreased dopaminergic neuron density or dopaminergic terminal density in the basal ganglia [2], compensated by increased dopamine receptor sensitization in the remaining dopaminergic neurons [3]. Levodopa (L-DOPA) treatment was expected to decrease involuntary movements in patients with LND; however, it exacerbated dystonia and hyperactivity in some patients [4].

Dopamine antagonists were also expected to diminish involuntary movements in patients with LND, and several studies showed dopamine antagonists attenuated self-injury in various animal models, including 6-hydroxydopamine-lesioned rats and monkeys with unilateral ventromedial tegmental lesions. However, it has been difficult to translate dopamine hypersensitivity into effective therapies for patients with LND, and results have varied. Other treatments are required to combat LND-related motor and behavioral dysfunction.

Glick [5] serendipitously discovered that S-adenosylmethionine (SAMe), administered to reduce transaminase levels elevated secondary to regular fentanyl use in a patient with LND, unexpectedly and dramatically improved self-injury behaviors and persisted over several years. Moreover, Chen et al. [6] reported the beneficial effect of SAMe on neurological symptoms, including dystonia and self-injury.

We encountered a patient with LND and severe dystonia, including abnormal laryngeal movements. After exhausting other therapeutic options, we administered SAMe treatment, and his neurological symptoms improved. This is a report of the patient's clinical course and a discussion of the effectiveness of SAMe treatment for LND. 


\section{Case Reports in Neurology}

\begin{tabular}{l|l}
\hline Case Rep Neurol 2019;11:256-264 \\
\hline DOI: 10.1159/000502568 & $\begin{array}{l}\text { (c) 2019 The Author(s). Published by S. Karger AG, Basel } \\
\text { www.karger.com/crn }\end{array}$
\end{tabular}

Momosaki et al.: The Effect of S-Adenosylmethionine Treatment on Neurobehavioral Phenotypes in Lesch-Nyhan Disease: A Case Report

\section{Case Report}

A 6-month-old boy presented to our institution with a 3-month history of motor development delay. He was the second child of healthy nonconsanguineous parents. The pre- and neonatal periods were uneventful. At admission, his height, weight, and head circumference were $68.8 \mathrm{~cm}$ ( -0.8 standard deviation [SD]), $7.8 \mathrm{~kg}(-0.5 \mathrm{SD})$, and $44.8 \mathrm{~cm}(+0.7 \mathrm{SD})$. By 6 months, his head control was poor, and he had abnormal dystonic movements, ballismus, and extensor spasms with marked hypotonia. The patient developed hyperuricemia (uric acid [UA], $10.3 \mathrm{mg} / \mathrm{dL}$ ) and increased urinary UA (urinary UA/creatinine ratio, 5.3 [control range $<2.0]$ ). Cerebrospinal fluid monoamine and related metabolite analyses were unremarkable (pyridoxal phosphate, $62.3 \mathrm{nmol} / \mathrm{L}$; pyridoxal, $58.0 \mathrm{nmol} / \mathrm{L} ; 5$-methyltetrahydrofolate, 61.0 $\mathrm{nmol} / \mathrm{L}$; homovanillic acid, $364.5 \mathrm{nmol} / \mathrm{L}$; 5-hydroxyindole acetic acid, $273.7 \mathrm{nmol} / \mathrm{L}$; 3-0methyldopa, $50.7 \mathrm{nmol} / \mathrm{L}$ ). Brain magnetic resonance imaging (MRI) showed no signs of cortical atrophy or abnormal intensities (Fig. 1a, b). His electroencephalogram was normal and his development quotient on the Enjoji Scale of Infant Analytical Development was 42 at 8 months of age. His blood T cells were HPRT negative because they were 6-thioguanine resistant. The genetic analysis of his HPRT gene revealed the hemizygous g.151C >T (p. R51X) mutation, and we diagnosed the patient with classic LND (Fig. 2). We administered allopurinol (up to $20 \mathrm{mg} / \mathrm{kg} /$ day) and added potassium citrate and sodium citrate when he was 15 months old. We added gabapentin (up to $40 \mathrm{mg} / \mathrm{kg} /$ day) for increased self-injury behavior at the age of 19 months and introduced SAMe treatment (up to $20 \mathrm{mg} / \mathrm{kg} /$ day) at the age of 23 months because his self-injury persisted.

The patient's self-injury gradually diminished after the introduction of SAMe. At 26 months of age, he showed stable head control and began picking up objects with each hand. His abnormal involuntary movements score was markedly decreased, especially for facial and oral movements (Table 1). However, he continued to demonstrate dyskinesias when he was angry or excited.

Although the patient had persistent paroxysmal inspiratory stridor resulting from abnormal laryngeal movement and frequent hospitalizations for bronchitis or bronchopneumonia before SAMe treatment, his laryngeal stridor resolved 6 months after SAMe treatment, and he has had few episodes of bronchitis or pneumonia since. MRI performed when he was 26 months old revealed no atrophy or abnormal brain intensities (including the basal ganglia; Fig. 1c, d). His (previously resolved) lower lip biting recurred when he was 34 months old. We administered risperidone (up to $0.05 \mathrm{mg} / \mathrm{kg} /$ day), and the patient's self-injury behavior completely resolved owing to the combination therapy of SAMe and risperidone. At this time, he is 4 years old and can move short distances by rolling.

\section{Discussion}

We present a boy with generalized dystonia and self-injury behavior secondary to LND. His recurrent self-injury behavior resolved after he received the combination of SAMe and risperidone treatment. Although he often experienced inspiratory stridor due to his laryngeal dystonia and had frequent episodes of aspiration pneumonitis and bronchitis, he was free of 


\section{Case Reports in Neurology}

Case Rep Neurol 2019;11:256-264

DOI: $10.1159 / 000502568$

2019 The Author(s). Published by S. Karger AG, Base www.karger.com/crn

Momosaki et al.: The Effect of S-Adenosylmethionine Treatment on Neurobehavioral Phenotypes in Lesch-Nyhan Disease: A Case Report

inspiratory stridor after SAMe treatment. At present, the patient still receives the combination SAMe (15-20 mg/kg/day) and risperidone $(0.05 \mathrm{mg} / \mathrm{kg} /$ day) treatment.

This patient has one of the most common mutations (g.151C>T [p. R51X]) that converts the codon encoding arginine to a stop signal [7]. This is a de novo mutation and was not detected when his parents underwent genetic analyses. In patients with LND, this defect is one of the most frequently occurring spontaneous mutations. This is because of the endogenous mutagenic potential of 5-methylcytosine deamination coupled with deoxyribonucleic acid (DNA) replication of the resulting T:G mismatch that produces a thymine transition mutation [8].

SAMe, a major methyl donor, influences central nervous system function through cellular transmethylation pathways, including the methylation of DNA, histones, protein phosphatase $2 \mathrm{~A}$, and several catecholamine moieties. SAMe has a prominent antidepressant effect, and some investigators reported a functional effect in mouse models of Alzheimer's disease, epilepsy, and amyotrophic lateral sclerosis. The pathophysiology of LND is not fully understood; however, it likely involves cellular adenosine depletion. SAMe may replenish the purine pool by serving as an adenosine donor. Adenosine can be formed into adenosine monophosphate by adenosine kinase and adenosine triphosphate. Adenylosuccinate lyase can form inosine monophosphate, which is then transformed to guanosine monophosphate by isocitrate dehydrogenase and replenishing guanosine triphosphate purines.

Chen et al. [6] reported the effectiveness of SAMe treatment for self-injury and dystonia in children with LND aged 1 month to 12 years. Conversely, Dolcetta et al. [9] reported that SAMe treatment improved self-injury in only 3 of 13 patients with LND who were aged 18 years or older and exacerbated self-injury in 5 patients. The effects of SAMe on LND-related self-injury and dopaminergic neurons probably depend on the patient's age.

Although the effectiveness of SAMe for the treatment of involuntary movements in neural diseases other than LND is controversial, it may produce effects in patients with basal ganglia lesions by modulating neurotransmitter release and increasing dopaminergic tone in brain regions, including the basal ganglia. More research is required to clarify the efficacy of SAMe for the treatment of involuntary movements caused by other neural diseases.

SAMe is a major methyl donor for transmethylation reactions, especially during synthesis of 2 myelin phospholipids: phosphatidylcholine and sphingomyelin. SAMe stimulates myelination in demyelinating diseases, such as methionine adenosyltransferase (MAT) I/III deficiency, and may help improve neurological symptoms in patients with MAT I/III deficiency [10]. On MRI, patients with LND show reduced volume of the basal ganglia, as seen in patients with dystonic movement disorders. At 26 months of age, our patient had no atrophic changes in the basal ganglia or other abnormal findings on MRI. SAMe treatment might stimulate myelination and prevent basal ganglia atrophy, thereby improving symptoms in patients with a dystonic movement disorder.

Risperidone is a D2 receptor antagonist with D1 receptor binding. The effect of risperidone on self-injury behavior varies among patients with LND [11]. The mechanism of risperidone for self-injury is controversial because risperidone has multiple targets within the central nervous system. SAMe appears to help replenish the purine pool in the brain and improves brain function. Risperidone balances those areas of the brain responsible for abnormal behaviors. Therefore, the SAMe and risperidone treatment could improve self-injury symptoms by different mechanisms. 


\section{Conclusion}

SAMe treatment alleviated generalized dystonia and self-injury behavior in our patient and appears to alleviate dystonic movements and improve quality of life in pediatric patients with LND. The combined treatment of SAMe and antipsychotics such as risperidone may be effective for self-injury behavior, which is uncontrolled by treatment with SAMe alone. Additional research is needed to determine the long-term safety and efficacy of SAMe and its appropriate dosage and combination with other medicines.

\section{Acknowledgements}

The authors thank the staff of the Kumamoto University Hospital Department of Pediatrics for their clinical contributions.

\section{Statement of Ethics}

The HPRT gene analysis was approved by the ethical committee of Tokyo Women's Medical University, and written informed consent was obtained from our patient's parents.

\section{Disclosure Statement}

The authors declare that there is no conflict of interest.

\section{Funding Sources}

This case report was supported, in part, by a Grant-in-Aid for Scientific Research from the Japanese Ministry of Education, Culture, Sports, Science, and Technology.

\section{Author Contributions}

Ken Momosaki and Jun Kido designed the case report and wrote the manuscript. Ken Momosaki, Jun Kido, Shiro Matsumoto, and Takaaki Sawada collected and analyzed data. Jun Kido, Shiro Ozasa, and Kimitoshi Nakamura supervised this case report and clinical practice. Atsuo Taniguchi performed the HPRT gene analysis, and Tomoyuki Akiyama performed the cerebrospinal fluid monoamine and related metabolite analyses. All authors read and approved the final manuscript. 


\section{References}

1 Torres RJ, Puig JG. Hypoxanthine-guanine phosophoribosyltransferase (HPRT) deficiency: Lesch-Nyhan syndrome. Orphanet J Rare Dis. 2007 Dec;2(1):48.

2 Wong DF, Harris JC, Naidu S, Yokoi F, Marenco S, Dannals RF, et al. Dopamine transporters are markedly reduced in Lesch-Nyhan disease in vivo. Proc Natl Acad Sci USA. 1996 May;93(11):5539-43.

3 Goldstein M, Anderson LT, Reuben R, Dancis J. Self-mutilation in Lesch-Nyhan disease is caused by dopaminergic denervation. Lancet. 1985 Feb;1(8424):338-9.

4 Visser JE, Schretlen DJ, Bloem BR, Jinnah HA. Levodopa is not a useful treatment for Lesch-Nyhan disease. Mov Disord. 2011 Mar;26(4):746-9.

5 Glick N. Dramatic reduction in self-injury in Lesch-Nyhan disease following S-adenosylmethionine administration. J Inherit Metab Dis. 2006 Oct;29(5):687.

6 Chen BC, Balasubramaniam S, McGown IN, O’Neill JP, Chng GS, Keng WT, et al. Treatment of Lesch-Nyhan disease with S-adenosylmethionine: experience with five young Malaysians, including a girl. Brain Dev. 2014 Aug;36(7):593-600.

7 Fu R, Ceballos-Picot I, Torres RJ, Larovere LE, Yamada Y, Nguyen KV, et al.; Lesch-Nyhan Disease International Study Group. Genotype-phenotype correlations in neurogenetics: Lesch-Nyhan disease as a model disorder. Brain. 2014 May;137(Pt 5):1282-303.

8 O'Neill JP, Finette BA. Transition mutations at CpG dinucleotides are the most frequent in vivo spontaneous single-based substitution mutation in the human HPRT gene. Environ Mol Mutagen. 1998;32(2):188-91.

9 Dolcetta D, Parmigiani P, Salmaso L, Bernardelle R, Cesari U, Andrighetto G, et al. Quantitative evaluation of the clinical effects of S-adenosylmethionine on mood and behavior in Lesch-Nyhan patients. Nucleosides Nucleotides Nucleic Acids. 2013;32(4):174-88.

10 Kido J, Sawada T, Momosaki K, Suzuki Y, Uetani H, Kitajima M, et al. Neonatal methionine adenosyltransferase I/III deficiency with abnormal signal intensity in the central tegmental tract. Brain Dev. 2019 Apr;41(4):382-8.

11 Crapper L, Ernst C. Comparative analysis of self-injury in people with psychopathology or neurodevelopmental disorders. Pediatr Clin North Am. 2015 Jun;62(3):619-31. 


\section{Case Reports in Neurology}
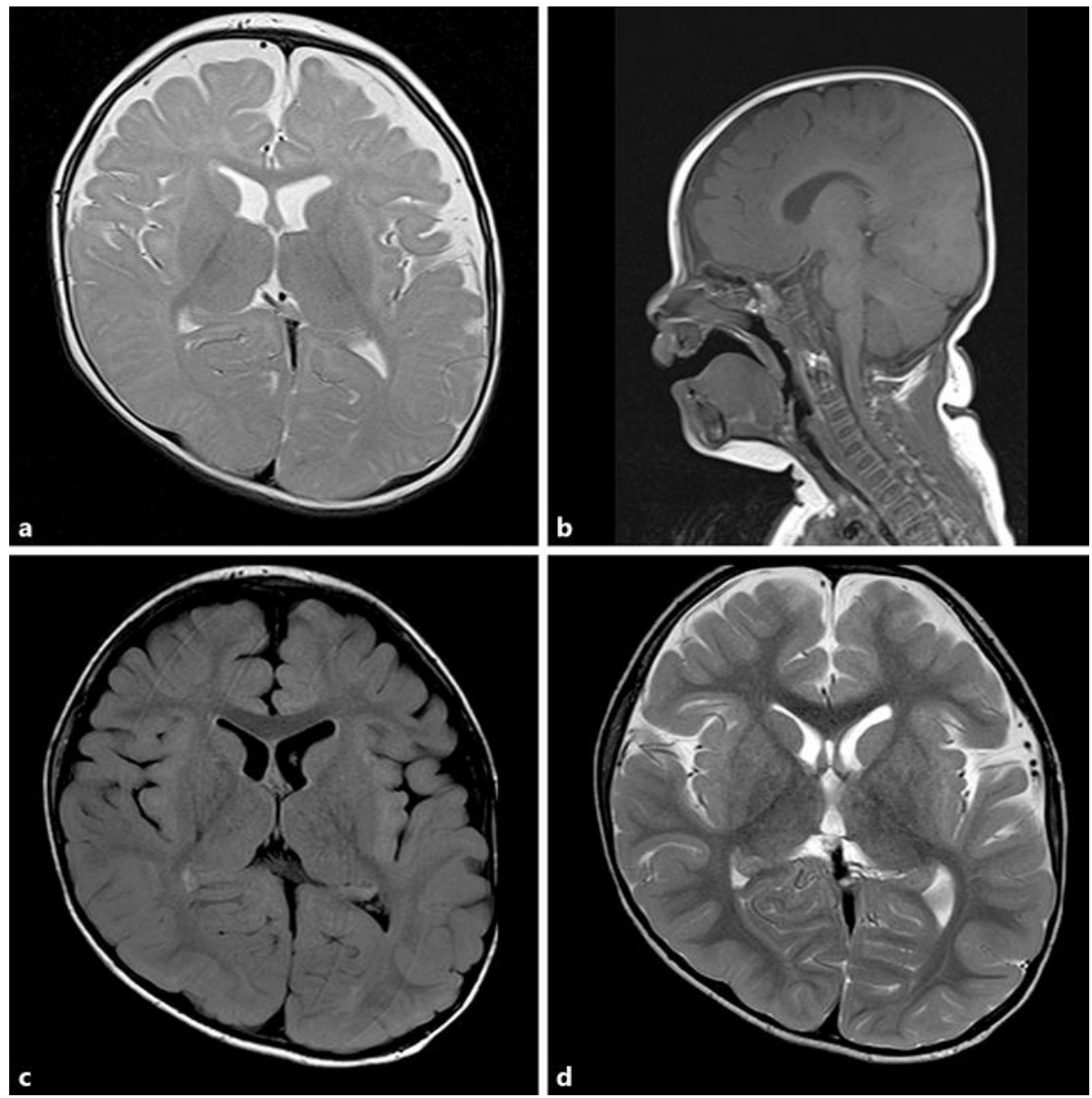

Fig. 1. Brain magnetic resonance imaging before $(\mathbf{a}, \mathbf{b})$ and after $(\mathbf{c}, \mathbf{d}) \mathrm{S}$-adenosylmethionine treatment in a boy with Lesch-Nyhan disease. a T2-weighted imaging at the age of 6 months. $\mathbf{b}$ T1-weighted imaging at the age of 6 months. c T1-weighted imaging at the age of 26 months. $\mathbf{d}$ T1-weighted imaging at the age of 26 months. 


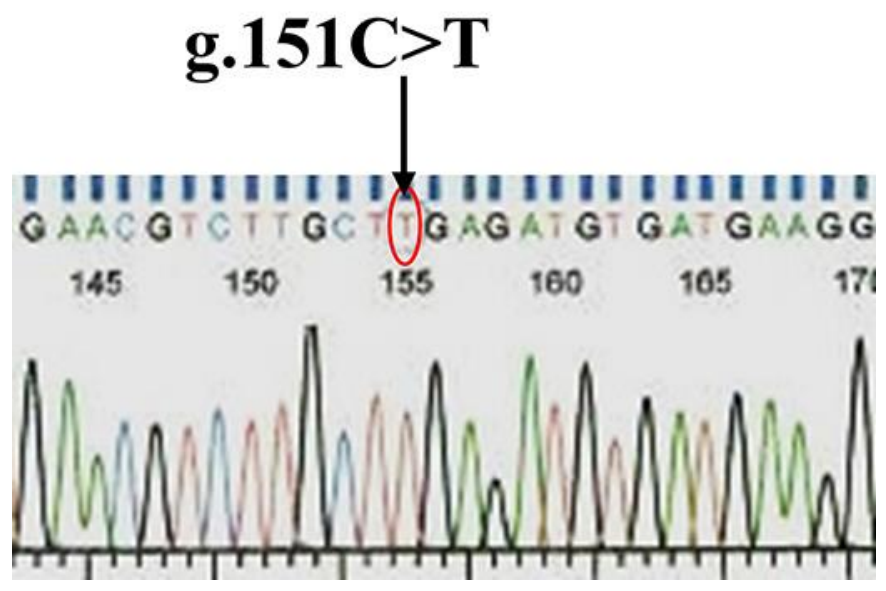

Fig. 2. The HPRT gene sequence analysis. The g.151C>T (p. R51X) mutation was a de novo mutation not detected in the HPRT genes of the patient's parents. 
Table 1. AIMS and BFMDRS before and after S-adenosylmethionine treatment

\begin{tabular}{llll}
\hline & Pre & $3 \mathrm{~m}$ & $6 \mathrm{~m}$ \\
\hline AIMS & & & \\
I: Facial and oral movements & 10 & 6 & 5 \\
II: Extremity movements & 8 & 5 & 5 \\
III: Trunk movements & 4 & 2 & 2 \\
IV: Global judgement & 10 & 8 & 7 \\
V: Dental status & n.p. & n.p. & n.p. \\
\hline AIMS total score & 32 & 21 & 19 \\
\hline BFMDRS & & & \\
Eyes & 1 & 0.5 & 0.5 \\
Mouth & 9 & 3 & 2 \\
Speech and swallow & 9 & 6 & 3 \\
Neck & 6 & 4 & 4 \\
Right arm & 12 & 9 & 9 \\
Left arm & 12 & 9 & 9 \\
Trunk & 16 & 12 & 12 \\
Right leg & 12 & 9 & 9 \\
Left leg & 12 & 9 & 9 \\
\hline BFMDRS total score & 89 & 61.5 & 57.5 \\
\hline
\end{tabular}

AIMS, Abnormal Involuntary Movement Scale; BFMDRS, Burke-FahnMarsden Dystonia Rating Scale; Pre, before S-adenosylmethionine treatment; $3 \mathrm{~m}$ and $6 \mathrm{~m}, 3$ months and 6 months after receiving Sadenosylmethionine; n.p., nothing particular. 